\title{
THE BOUNDARY OF CONVEX FUNDAMENTAL DOMAINS OF FUCHSIAN GROUPS
}

\author{
Peter J. Nicholls and Peter L. Waterman
}

\section{Introduction}

Let $\Delta$ denote the unit disc of the complex plane endowed with metric $\rho$ derived from the differential

$$
d \rho=\frac{2|d z|}{1-|z|^{2}} .
$$

If $\Gamma$ is a discrete group preserving $\Delta$ and $a \in \Delta$ is not fixed by any non-identity transform of $\Gamma$, then we may construct the Dirichlet region centered at $a$, denoted $D_{a}$, as follows

$$
D_{a}=\{z \in \Delta: \rho(z, a)<\rho(z, \gamma(a)) \text { for all } \gamma \in \Gamma \backslash I\}
$$

A point $\xi \in \partial \Delta$ is said to be represented on the boundary of $D_{a}$ if there exists $\gamma \in \Gamma$ with $\gamma(\xi) \in \partial D_{a}$. It is well known [1] that every parabolic fixed point and every ordinary point in $\partial \Delta$ must be represented on the boundary of every Dirichlet region and we are led to define the Dirichlet set, denoted $D$, as follows

$$
D=\left\{\xi \in \partial \Delta: \text { for each } a \in \Delta, \text { there exists } \gamma \in \Gamma \text { with } \gamma(\xi) \in \partial D_{a}\right\}
$$

At the other end of the spectrum are horocyclic limit points. A point $\xi \in \partial \Delta$ is said to be a horocyclic limit point for $\Gamma$ if the orbit of one, and hence every, point of $\Delta$ enters every horocycle at $\xi$. It is not too difficult to prove [1] that a horocyclic limit point cannot be represented on the boundary of any Dirichlet region for $\Gamma$. Writing $H$ for the set of horocyclic limit points we thus have

$$
\partial \Delta=D \cup H \cup G
$$

a disjoint union, where $G$ may be defined as the complement of $D \cup H$ in $\partial \Delta$. The set $G$ is called the Garnett set and may be characterized geometrically as follows. If $\xi \in \partial \Delta$, then $\xi \in G$ if and only if there exists $a \in \Delta$ and a horocycle $h$ at $\xi$ with the property that no image of $a$ is interior to $h$ but infinitely many images of $a$ are interior to any horocycle containing $h$ in its interior. Thus the orbit of $a$ clusters around the critical horocycle $h$. The set $G$ first arose in the work of Sullivan [6] on the ergodic properties of discrete groups. Sullivan showed that $G$ 
has zero measure but the first author showed that, in general, it is not empty [3]. The method used to construct a point of $G$ was to find a point $\xi$ represented on the boundary of some domain $D_{a}$ but not represented on the boundary of $D_{b}$ say. Since this point is not represented on the boundary on $D_{b}$, it cannot possibly belong to $D$. Also, its representation on $\partial D_{a}$ prohibits it from being in $H$. A point with these properties exhibits the Garnett behavior with respect to the orbit of $b$, but not with respect to the orbit of $a$.

It is perhaps easier to visualize this behavior in the upper half-plane. In order that $\infty$ be Garnett for a group $\Gamma$ preserving the upper half-plane it is necessary and sufficient that some orbit be of bounded height with every point in this orbit having an image of greater height. In this case the Ford set, obtained by choosing a point of greatest height from each orbit, will not be a fundamental set for $\Gamma$.

Of course, another way for a point to belong to $G$ is for it to exhibit the Garnett behavior with respect to every orbit-such a point will be called a universal Garnett point. In order that $\infty$ be a universal Garnett point for a group $\Gamma$ preserving the upper half-plane it is necessary and sufficient that every orbit be of bounded height and that every point in the upper half-plane have an image with greater height.

We thus have a nice classification of points of $\partial \Delta$-those in $H$, and universal Garnett points, cannot appear on the boundary of any Dirichlet region. All other points are represented on the boundary of at least one such region and, in the case of Dirichlet points, of all such regions.

Our object in this paper is to consider the analoguous situation as it applies to the wider class of convex, locally finite domains. This is the largest class of domains usually considered - these are the ones for which the quotient domain is a Riemann surface which is conformally equivalent to the disc modulo the group [1]. We aim to find which points of $\partial \Delta$ can be represented on the boundary of a convex, locally finite domain. In
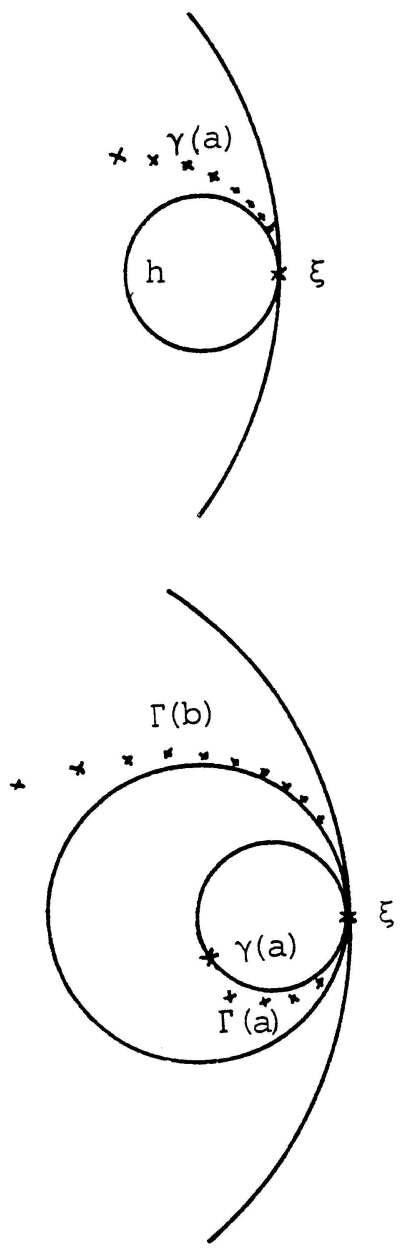
one sense we find a very simple answer in that an obviously necessary geometric condition turns out to be sufficient. Relating this geometric condition to the usual classification of limit points is not simple and we do not have a definitive classsification. We have some results along these lines and several examples that illustrate the complications which may arise. 
We conclude this section by giving an example to show that universal Garnett points exist, and indeed can lie on the boundary of a fundamental domain.

Theorem 1.1. There exists a Fuchsian group $\Gamma$ with a convex, locally finite fundamental domain having a universal Garnett point on its boundary.

Proof. For $n=1,2,3, \ldots$ define a Möbius transform

$$
E_{n}=\left(\begin{array}{cc}
1-1 / n^{3} & -(n !)^{3}\left[1+\left(1-1 / n^{3}\right)^{2}\right] \\
1 /(n !)^{3} & -\left(1-1 / n^{3}\right)
\end{array}\right)
$$

and set $\Gamma=\left\langle\left\{E_{n}\right\}\right\rangle, n \geq 2$. We first need to show that $\Gamma$ is a discrete group and we do this by exhibiting a non empty packing for the action of $\Gamma$ on the upper half-plane. For each $n$ let $C_{n}$ be the circle centered on the real axis and intersecting this axis at the points $3(n !)^{3}$ and $E_{n}\left(3(n !)^{3}\right)$. Note that $E_{n}$ is an elliptic transform of order two and so preserves the circle $C_{n}$.

Now

$$
\begin{aligned}
E_{n}\left(3(n !)^{3}\right) & =(n !)^{3}\left[\frac{3\left(1-1 / n^{3}\right)-1-\left(1-1 / n^{3}\right)^{2}}{\left(\left(3-\left(1-1 / n^{3}\right)\right)\right.}\right] \\
& <(n !)^{3}\left(1-1 / n^{3}\right)=E_{n}(\infty)<3(n !)^{3}
\end{aligned}
$$

and so $E_{n}(\infty)$ is interior to the circle $C_{n}$. It follows that, for each $n, E_{n}$ maps the exterior of $C_{n}$ onto its interior. We now check the relative placement of the circles $C_{n}$. We observe that

$$
\begin{aligned}
E_{n}\left(3(n !)^{3}\right) & =3((n-1) !)^{3}\left[\frac{\left(1-1 / n^{3}\right) n^{3}-\frac{1}{3} n^{3}\left[1+\left(1-1 / n^{3}\right)^{2}\right]}{3-\left(1-1 / n^{3}\right)}\right] \\
& =3((n-1) !)^{3}\left[\frac{n^{3}-1-1 / n^{3}}{3\left(2+1 / n^{3}\right)}\right]>3[(n-1) !]^{3} \quad \text { for all } n \geq 2 .
\end{aligned}
$$

and so the circles $C_{n}$ are exterior to each other. It is now immediate that the region exterior to all $C_{n}, n=2,3, \ldots$, is a packing for $\Gamma$ which is thus a discrete group. We see immediately that

$$
E_{n}\left(3(n !)^{3}\right) \sim \frac{1}{2}(n !)^{3}
$$

and, using a result of Maskit [2, p. 172], can conclude that the region exterior to all $C_{n}$ is a fundamental domain for $\Gamma$. This fundamental region is clearly convex, locally finite, and has $\infty$ on its boundary. We will show that $\infty$ is a universal Garnett point. As a first step we note that, for any $z$ in the upper half-plane and for any $n$

$$
\operatorname{Im}\left(E_{n}(z)\right)=\frac{\operatorname{Im}(z)}{\left|z /(n !)^{3}-\left(1-1 / n^{3}\right)\right|^{2}} .
$$


For each $z$ we may find an $n$ such that

$$
\left|\frac{z}{(n !)^{3}}-\left(1-\frac{1}{n^{3}}\right)\right|<1
$$

and so every $z$ has a $\Gamma$ image of larger imaginary part. It follows that $\infty$ is either a universal Garnett point or is a horocyclic limit point. To rule out the latter possibility we need to show that the set

$$
\{\operatorname{Im} V(i): V \in \Gamma\}
$$

is bounded. For this we need some preliminary estimates.

Suppose an integer $n(>2)$ is fixed, that $k$ is an integer less than $n$ and that $z$ is interior to $C_{k}$. It is easily checked that

$$
\left|\frac{z}{(n !)^{3}}-\left(1-\frac{1}{n^{3}}\right)\right| \geq 1-4 / n^{3} \geq 1 / 2 .
$$

Similarly it can be shown that if $k$ is greater than $n$ and $z$ is interior to $C_{k}$ then

$$
\left|\frac{z}{(n !)^{3}}-\left(1-\frac{1}{n^{3}}\right)\right| \geq \frac{1}{2} n^{3} \geq 4 \text {. }
$$

We note that $i$ is exterior to all the circles $C_{n}$ and that

$$
\operatorname{Im}\left(E_{n}(i)\right) \leq 1 /\left(1-1 / 2^{3}\right)^{2}<\frac{3}{2} .
$$

If $V \in \Gamma$ we write $V$ as a reduced word in the generators

$$
V=E_{n_{k}} \cdots E_{1} E_{n_{0}}
$$

and note that from (1.1), (1.2), and (1.3) above and an induction on the formula

$$
\operatorname{Im}\left(\frac{a z+b}{c z+d}\right)=\frac{\operatorname{Im}(z)}{|c z+d|^{2}}
$$

we have

$$
\operatorname{Im} V(i)=\operatorname{Im}\left(E_{n_{k}} \cdots E_{n_{0}}(i)\right) \leq \frac{\operatorname{Im} E_{n_{0}}(i)}{\prod_{i=2}^{k+2}\left(1-4 / i^{3}\right)^{2}} \leq \frac{3 / 2}{\prod_{i=2}^{\infty}\left(1-4 / i^{3}\right)^{2}}<\infty
$$

and so $\infty$ is not a horocyclic limit point. It follows that $\infty$ is a universal Garnett point for $\Gamma$ and the proof of the theorem is complete. 


\section{A geometric condition}

If $\xi \in \partial \Delta$ lies on the boundary of a convex fundamental domain $D$ for a discrete group $\Gamma$ then it is clear that we may find a half-geodesic $\sigma$ ending at $\xi$ which meets none of its $\Gamma$-images-we merely join $\xi$ to an interior point of $D$. If $\xi$ lies on the boundary of a convex, locally finite fundamental domain $D$ then we find a half-geodesic $\sigma$ as above and note that the images of $\sigma$ cannot accumulate in $\Delta$ (else $D$ is not locally finite). It follows then that for any $z \in \Delta$ the set

$$
\{\rho(V(z), \sigma): V \in \Gamma\}
$$

has no finite accumulation point. Thus no orbit can approach $\xi$ within a cone. We recall [4] the definition of a conical limit point and we have shown that if $\xi$ lies on the boundary of a convex, locally finite fundamental domain then

(i) $\xi$ is not a conical limit point and

(ii) there is a half-geodesic ending at $\xi$ which meets none of its $\Gamma$-images.

The following result shows that the second property above is a property of $\xi$ and not of any particular half-geodesic.

Theorem 2.1 Let $\Gamma$ be a Fuchsian group and suppose $\xi \in \partial \Delta$ is not a conical limit point. If some half-geodesic ending at $\xi$ meets none of its $\Gamma$-images, then every half-geodesic ending at $\xi$ meets only finitely many of its $\Gamma$-images.

Proof. We conjugate to the upper halfplane and assume that $\xi=\infty$. We suppose that the set

$$
\sigma=\{z=i y: y>1\}
$$

meets infinitely many images under $\Gamma$. Under the assumption that $\infty$ is not a conical limit point we show that any geodesic segment

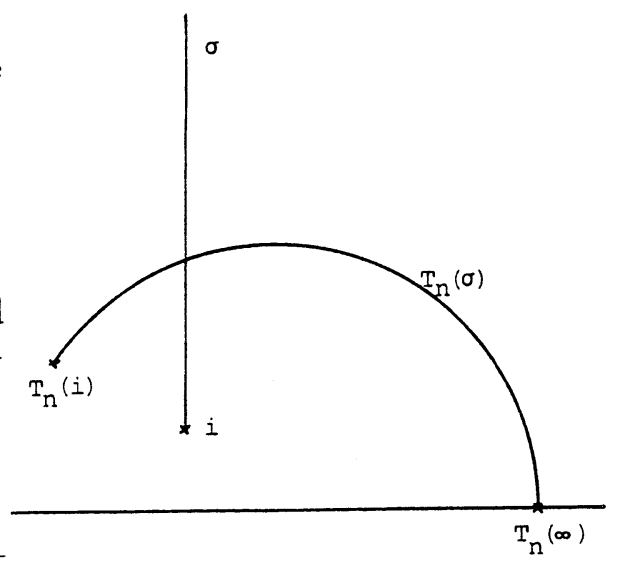

meets infinitely many of its group images-

$$
\sigma_{a, b}=\{z=a+i y: y>b\}
$$

this will suffice to prove the theorem.

Suppose then that $\left\{T_{n}: n=1,2,3, \ldots\right\} \subset \Gamma$ and $T_{n}(\sigma) \cap \sigma \neq \emptyset$ for every $n$. Our first observation is that if $\left\{T_{n}(\infty)\right\}$ is a bounded set of reals then $\left\{\rho\left(T_{n}(\sigma), i\right)\right\}$ is bounded and hence so is $\left\{\rho\left(\sigma, T_{n}^{-1}(i)\right)\right\}$. Thus the orbit of $i$ approaches $\infty$ in a cone which contradicts the fact that $\infty$ is not a conical limit point. Thus we assume, without loss of generality-on a subsequence if necessary,

$$
T_{n}(\infty) \rightarrow+\infty
$$


With this assumption we next show that $\operatorname{Re}\left(T_{n}(i)\right) \rightarrow-\infty$. Consider the picture and it is clear that $\operatorname{Re}\left(T_{n}(i)\right)<0$ for every $n$. If $\left\{\operatorname{Re}\left(T_{n}(i)\right): n=\right.$ $1,2, \ldots\}$ is bounded then (on a subsequence possibly) either

$$
T_{n}(i) \rightarrow \infty \text { in a chimney } \quad-k<\operatorname{Re}(z)<0
$$

which makes $\infty$ a conical limit point or

$$
T_{n}(i) \rightarrow x
$$

for some non-positive real number $x$. However, in this latter case, $i$ remains a bounded distance from $T_{n}(\sigma)$ and we argue as before to show that this makes $\infty$ a conical limit point. We have shown

$$
\operatorname{Re}\left(T_{n}(i)\right) \rightarrow-\infty
$$

We next wish to show that $\rho\left(T_{n}(i)\right.$, imaginary axis $) \rightarrow \infty$ as $n \rightarrow \infty$. If this were not the case then the set $\left\{T_{n}(i): n=1,2, \ldots\right\}$ would be contained in some conical region around the imaginary axis as shown below.

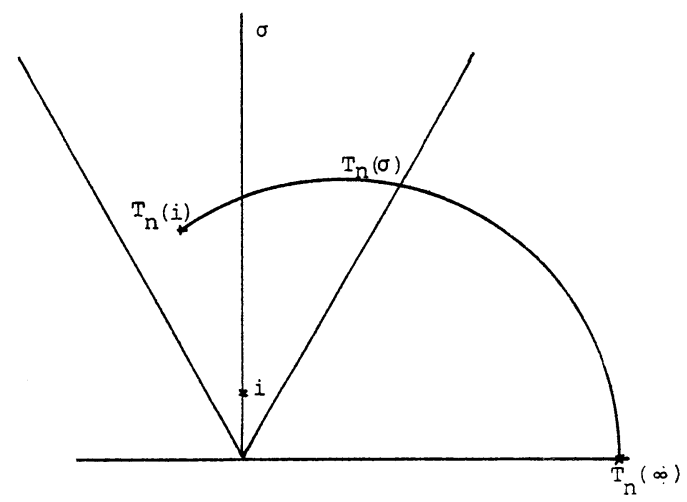

This again implies that $\infty$ is a conical limit point and thus we have

$$
\rho\left(T_{n}(i) \text { Imaginary axis }\right) \rightarrow \infty \quad \text { as } n \rightarrow \infty .
$$

Choose $a+i b$ in the upper half-plane and write $\sigma_{a, b}$ for the half-geodesic

$$
\sigma_{a, b}=\{z=a+i y: y>b\}
$$




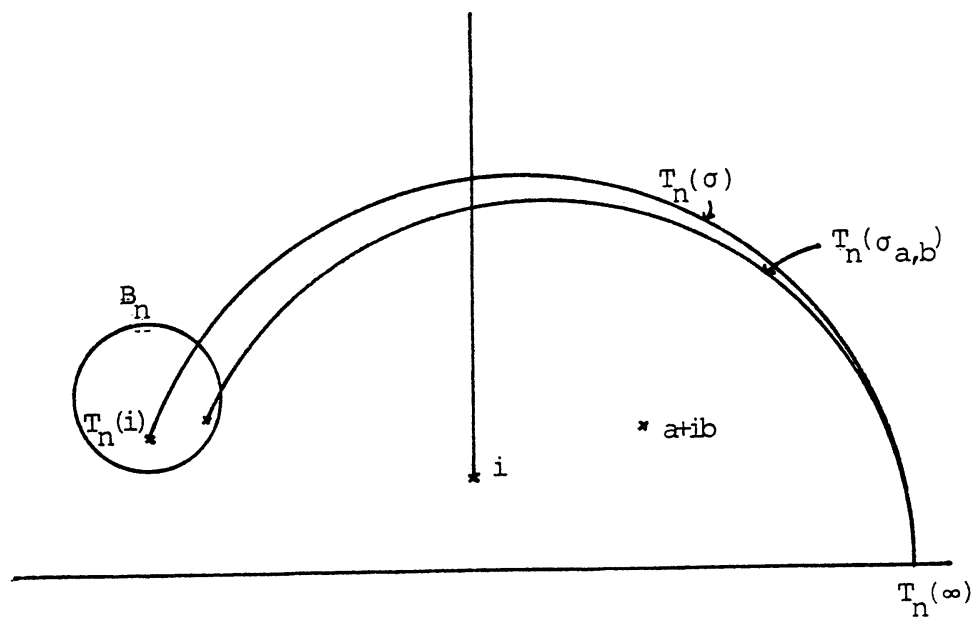

and let $k=\rho(i, a+i b)$. Write $B_{n}$ for the hyperbolic ball centered at $T_{n}(i)$ and of radius $2 k$-this ball clearly contains $T_{n}(a+i b)$. From $(2.1)$ we note that for $n$ large enough

$$
T_{n}(\infty)>a
$$

From (2.2) and (2.3) we know that for $n$ large enough $\sigma_{a, b} \cap B_{n}=\emptyset$. Clearly then, for all $n>N$ say we will have the situation illustrated above and it is clear that $T_{n}\left(\sigma_{a, b}\right) \cap \sigma_{a, b} \neq \emptyset$ and the proof of the theorem is complete.

What this theorem says is that if $\xi$ is not conical and if some half-geodesic ending at $\xi$ is simple (meets none of its $\Gamma$-images ) then every half-geodesic ending at $\xi$ contains a simple half-geodesic. These considerations motivate the following definition.

Definition. For a Fuchsian group $\Gamma$ acting in $\Delta$ a point $\xi$ of $\partial \Delta$ is said to be simple if it is not a conical limit point and if some half-geodesic ending at $\xi$ is simple.

Our next result shows that a simple point must be one end of a simple geodesic.

Theorem 2.2. Let $\Gamma$ be a Fuchsian group acting in $\Delta$ and suppose that $\xi \in \partial \Delta$ is a simple point for $\Gamma$ then there exists a complete geodesic $\eta$ ending at $\xi$ which meets none of its $\Gamma$-images.

Proof. Consider a half-geodesic $\sigma$, ending at $\xi$, which is simple. Choose $V \in \Gamma$ and note that the set $\sigma \cup V(\sigma)$ meets none of its $\Gamma$ images (we assume $V(\xi) \neq \xi$ ). Join the finite ends of $\sigma$ and $V(\sigma)$ by a geodesic segment $\tau$ so that $\xi$ is joined to $V(\xi)$ by a piecewise

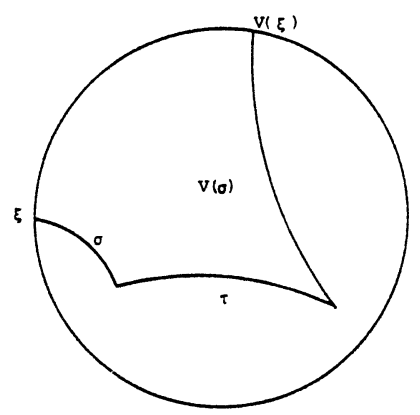


geodesic path- $\sigma \cup \tau \cup V(\sigma)$-as shown at the right.

Call this path $l$ and note that $l$ is not necessarily simple. However, we do claim that $l$ meets only finitely many of its $\Gamma$-images. If this were not the case then, by our assumption on $\sigma$, we would have infinitely many $\Gamma$-images of $\sigma$ meeting $\tau$. But this would make $\xi$ a conical limit point contrary to hypothesis. If we project $l$ to the quotient surface we obtain a piecewise geodesic with a finite number of loops in it. Deleting the loops and lifting back up to the disk we obtain a subset $L$ of $l$ which comprises $\sigma, V(\sigma)$, and a finite collection of subintervals of $\tau$ and which is simple. From our construction, an end point of one subinterval of $\tau$ is equivalent under $\Gamma$ to the nearest end point of the next subinterval. The diagram at the right shows the situation.

If, with the notation of the picture, the piece of $L$ to the right of $z_{1}$ is replaced by its $V_{1}^{-1}$ image then the resulting set is still simple and has one less "gap". Repeating this operation finitely often yields a simple piecewise geodesic path $L^{\prime}$ say, from $\xi$ to some $S(\xi)$ as shown the right. Let $\eta$ be the geodesic joining $\xi$ to $S(\xi)$.

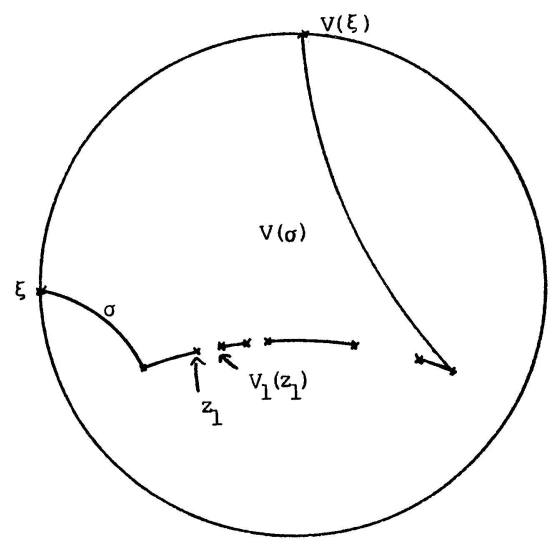

We now show that $\eta$ is simple and this will complete the proof of the theorem. But this is easy. If $\eta$ intersects $T(\eta)$, say, then the geodesic from $T(\xi)$ to $T S(\xi)$ crosses $\eta$ and this forces $T\left(L^{\prime}\right)$ to cross $L^{\prime}$ in contradiction to the above. The proof of the above theorem may easily be strengthened to give:

Theorem 2.3. Let $\Gamma$ be a Fuchsian group acting in $\Delta$ and $\xi, \eta \in \partial \Delta$ simple points then the following are equivalent

(i) for some $T \in \Gamma$ the geodesic with endpoints $\xi, T \eta$ is simple;

(ii) for some half-geodesics $\sigma_{1}, \sigma_{2}$ ending at $\xi, \eta$ respectively only finitely many $\Gamma$-images of $\sigma_{1}$ cross $\sigma_{2}$.

We can now prove the main theorem of the section.

Theorem 2.4. Let $\Gamma$ be a Fuchsian group acting in $\Delta$ and $\xi \in \partial \Delta$. In order that $\xi$ be on the boundary of some convex, locally finite fundamental domain for 
$\Gamma$ it is necessary and sufficient that $\xi$ be simple.

Proof. From our comments at the beginning of this section the necessity of the condition follows. Thus we assume $\xi$ is simple and will construct a convex, locally finite fundamental domain $D$ for $\Gamma$ which has $\xi$ on its boundary.

Let $\eta$ be the simple geodesic ending at $\xi$ whose existence is guaranteed by Theorem 2.2. Note that the other end of $\eta$ is an image of $\xi$ and is thus also a simple point. Define the set $D$ as follows.

$$
D=\{z \in \Delta: \rho(z, \eta)<\rho(z, V(\eta)) \text { for all } V \in \Gamma \backslash I\}
$$

For any $V \in \Gamma \backslash I, V(\eta) \cap \eta=\emptyset$. The bisector of $V(\eta)$ and $\eta$ is a geodesic dividing $\Delta$ into two half-planes, one of which contains $\eta$. Call this half-plane $H_{V}$.

Clearly $D=\cap_{V \in \Gamma} H_{V}$ which is a convex set containing $\eta$, and hence with $\xi$ on its boundary. It is evident from the definition that $D$ contains no two points which are equivalent under $\Gamma$ and is thus a convex packing. We next show that $D$ is a covering. If this is not the case then there exists $z \in \Delta$ which has no closest image of $\eta$. There will then exist a sequence $\left\{V_{n}\right\} \subset \Gamma$ such that

$$
\rho\left(z, V_{n}(\eta)\right) \rightarrow k<\infty
$$

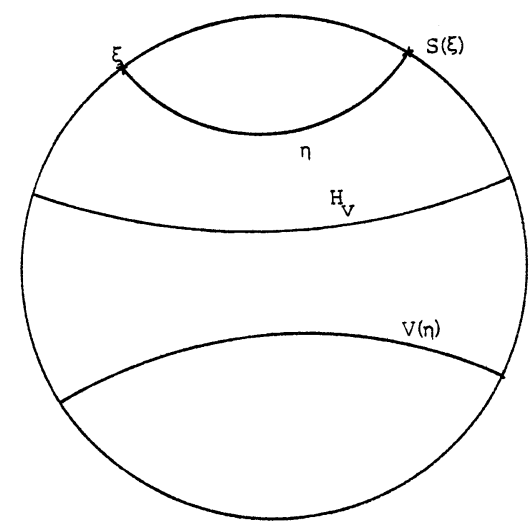

and so the $V_{n}^{-1}$ images of $z$ will remain a bounded distance from $\eta$. In this situation $V_{n}^{-1}(z)$ will approach $\xi$ (or $S(\xi)$ ) in a cone contradicting the fact that $x$ is not conical. We have shown that $D$ is a convex fundamental domain with $\xi$ on its boundary. It remains only to show that $D$ is locally finite. If this is not the case then there exists $z \in \Delta$ at which $\Gamma$-images of $D$ accumulate. But this cannot happen since $z$, as remarked above, must have a closest image of $\eta$.

In contrast to the situation for ordinary points and parabolic fixed points which are necessarily represented on the boundary of a convex locally finite fundamental domain:

Theorem 2.5. There exists a Fuchsian group $\Gamma$ and two simple points which cannot both be represented on the boundary of the same fundamental domain.

Proof. We construct an example of a surface with a geodesic $\sigma=\sigma_{1} \cup \sigma_{2}$ such that $\sigma$ crosses itself infinitely often but the half-geodesics $\sigma_{1}$ and $\sigma_{2}$ are simple. The theorem then follows from Theorem 2.3. 
The basic "building block" is obtained by glueing alternate sides of two regular all-right octagons:

Note the existence of the geodesic segments $\sigma_{1}, \sigma_{2}$ orthogonal to the boundaries shown.

Assembling copies of this building block we get:
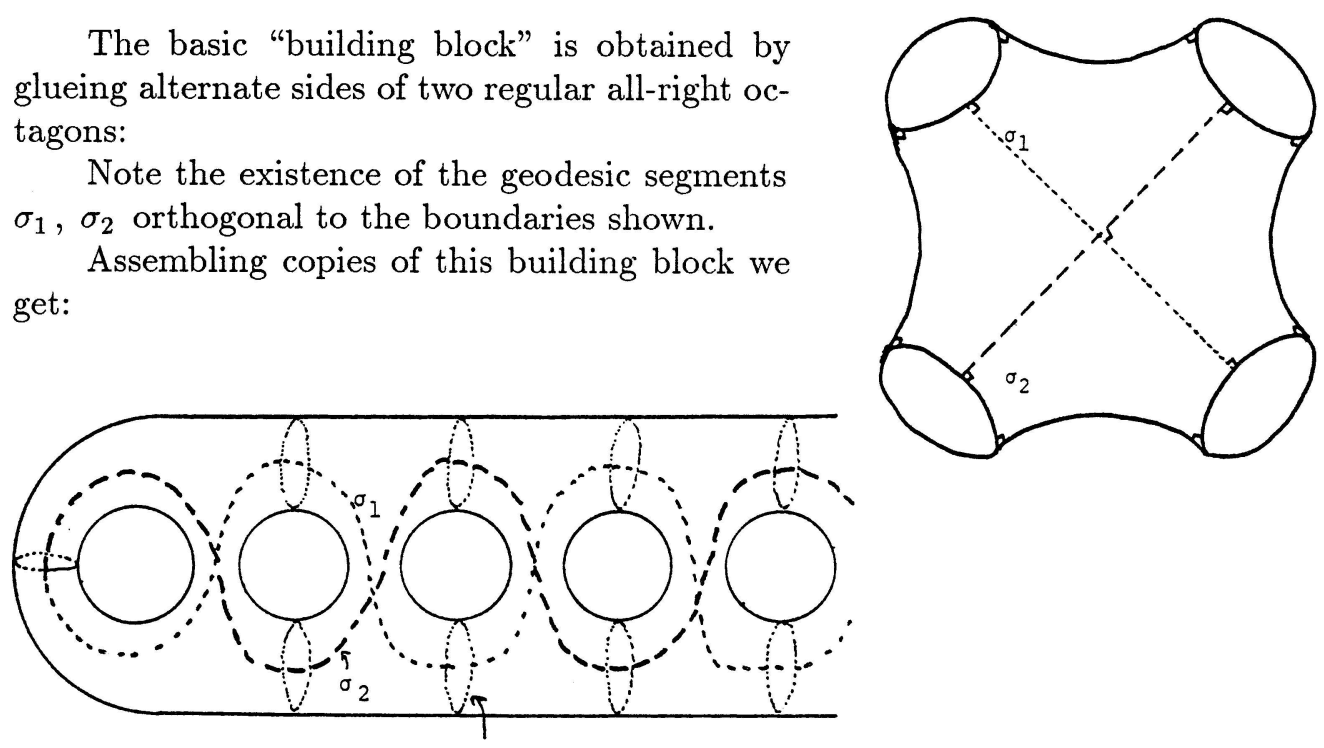

Boundary curves of building blocks

The theorem follows after observing that $\sigma_{1}, \sigma_{2}$ eventually leave any compact set so that they lift to half-geodesics which do not end at conical limit points.

\section{The set of simple points}

Our aim in this section is to consider the relationship between the set of simple points and the other subclasses of the limit set with which we have been concerned. We first need a condition which will enable us to recognize horocyclic limit points. Throughout this section we will be working in the upper half-plane model of hyperbolic space.

Lemma 3.1. Let $\Gamma$ be a Fuchsian group preserving the upper half-plane. If $\Gamma$ contains a sequence $\left\{T_{n}: n=1,2, \ldots\right\}$ with

$$
T_{n}(z)=\frac{a_{n} z+b_{n}}{c_{n} z+d_{n}} \quad a_{n} d_{n}-b_{n} c_{n}=1
$$

and

(i) $a_{n} \rightarrow a: 0 \leq a<1$ and

(ii) $c_{n} \rightarrow 0 \quad\left(c_{n} \neq 0\right)$

then $\infty$ is a horocyclic limit point.

Proof. Consider

$$
T_{n} T_{m}=\frac{\left(a_{n} a_{m}+b_{n} c_{m}\right) z+(\quad)}{\left(c_{n} a_{m}+d_{n} c_{m}\right) z+(\quad)} .
$$


For each $n$ choose $m$ so large that $\left|b_{n} c_{m}\right|<1 / n$ and $\left|d_{n} c_{m}\right|<1 / n$. Thus we have a sequence ${ }_{1} T_{n}(z)=\left({ }_{1} a_{n} z+{ }_{1} b_{n}\right) /\left({ }_{1} c_{n} z+{ }_{1} d_{n}\right)$ of elements of $\Gamma$ with ${ }_{1} a_{n} \rightarrow a^{2}$ and ${ }_{1} c_{n} \rightarrow 0$. Repeating the process we find a sequence $\left\{S_{n}\right\}$ of elements of $\Gamma$ with

$$
S_{n}(z)=\frac{\alpha_{n} z+\beta_{n}}{\gamma_{n} z+\delta_{n}}
$$

and $\alpha_{n} \rightarrow 0, \gamma_{n} \rightarrow 0$. Recalling that

$$
\operatorname{Im} S_{n}(z)=\frac{\operatorname{Im} z}{\left|\gamma_{n} z+\delta_{n}\right|^{2}}
$$

we note that $\operatorname{Im}\left(S_{n}(z)\right) \rightarrow \infty$ and so $\infty$ is a horocyclic limit point as required.

We will consider how the set of simple points relates to the sets $D, G, H$ introduced in Section 1.

Theorem 3.2. Let $\Gamma$ be a Fuchsian group preserving the upper half plane. If $\xi \in D \cup G$ then $\xi$ is simple.

Proof. If $\xi \in D \cup G$ and is not a universal Garnett point then $\xi$ must appear on the boundary of some Dirichlet domain. Such a domain is convex and locally finite so, by Theorem $2.3, \xi$ is a simple point. It remains only to deal with the case where $\xi$ is a universal Garnett point.

We assume that $\infty$ is a universal Garnett point and write

$$
\sigma=\{z: z=i y, y>1\}
$$

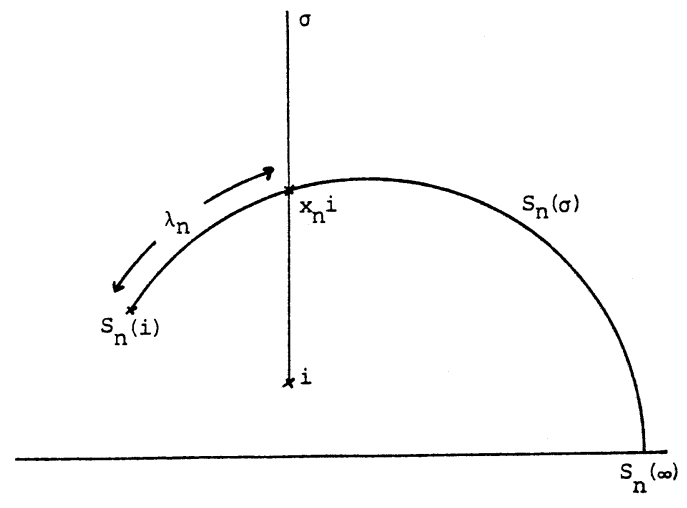

-a half-geodesic ending at $\infty$. We

will show that $\sigma$ meets only finitely many of its $\Gamma$-images and the proof will be completed.

Assume then that $\sigma$ meets infinitely many of its images, say $S_{n}(\sigma)$. Write

$$
x_{n} i=\sigma_{\cap} S_{n}(\sigma), \quad \lambda_{n}=\rho\left(S_{n}(i), x_{n} i\right) .
$$

With the assumption that $\infty$ is not a conical limit point we note that $x_{n} \rightarrow$ $\infty$ and $\lambda_{n} \rightarrow \infty$. If we take the $S_{n}^{-1}$ image of this picture then $S_{n}^{-1}\left(x_{n} i\right)=$ $\sigma \cap S_{n}^{-1}(\sigma)=x_{n}^{\prime}$ say and we have $x_{n}^{\prime} \rightarrow \infty$. We may suppose without loss of generality that $x_{n}^{\prime} \geq x_{n}$ for all $n$ (this will merely be a matter of relabelling). Writing

$$
S_{n}(z)=\frac{a_{n} z+b_{n}}{c_{n} z+d_{n}}
$$


then

$$
x_{n}^{\prime} i=\frac{d_{n} x_{n} i-b_{n}}{-c_{n} x_{n} i+a_{n}}=\frac{-\left(a_{n} b_{n}+c_{n} d_{n} x_{n}^{2}\right)+x_{n} i}{c_{n}^{2} x_{n}^{2}+a_{n}^{2}}
$$

from which

$$
c_{n}^{2} x_{n}^{2}+a_{n}^{2} \leq 1
$$

and

$$
a_{n} b_{n}+c_{n} d_{n} x_{n}^{2}=0
$$

Thus

$$
d_{n}\left(a_{n}^{2}+c_{n}^{2} x_{n}^{2}\right)=a_{n} .
$$

From (3.1) we have $c_{n} \rightarrow 0$ and $\left|a_{n}\right| \leq 1$, but there can be no subsequence on which $a_{n}$ converges to a number less than one-otherwise, by Lemma $3.1, \infty$ is a horocyclic limit point. Thus we may assume

$$
c_{n} \rightarrow 0, \quad a_{n} \rightarrow 1, \quad d_{n} \rightarrow 1
$$

and, by (3.2),

$$
a_{n} \leq d_{n} \quad \text { and } \quad a_{n} d_{n}<1 .
$$

and so we have

$$
b_{n} c_{n}<0
$$

We calculate

$$
\begin{aligned}
{\left[\operatorname{Im}\left(S_{n}^{-2}(\sigma) \cap \sigma\right)\right]^{2} } & =\frac{-b_{n}\left(a_{n}^{2}+b_{n} c_{n}\right)}{c_{n}\left(d_{n}^{2}+b_{n} c_{n}\right)}<\frac{-b_{n}}{c_{n}}\left(\frac{a_{n}}{d_{n}}\right)^{2} \\
& \leq \frac{-b_{n} a_{n}}{c_{n} d_{n}}=\left[\operatorname{Im}\left(S_{n}^{-1}(\sigma) \cap \sigma\right)\right]^{2}
\end{aligned}
$$

provided $n$ is large enough. The hyperbolic distance between

$$
S_{n}^{-2}(\sigma) \cap \sigma \quad \text { and } \quad S_{n}^{-1}(\sigma) \cap \sigma
$$

is

$$
\int_{u}^{v} \frac{d t}{t}
$$


where $v^{2}=\left(-b_{n} a_{n}\right) /\left(c_{n} d_{n}\right)$ and $u^{2}=-b_{n}\left(a_{n}^{2}+b_{n} c_{n}\right) / c_{n}\left(d_{n}^{2}+b_{n} c_{n}\right)$. Thus the distance is

$$
\frac{1}{2} \ln \left[\frac{a_{n}\left(d_{n}^{2}+b_{n} c_{n}\right)}{d_{n}\left(a_{n}^{2}+b_{n} c_{n}\right)}\right]
$$

which converges to zero as $n \rightarrow \infty$. We have shown that as $n \rightarrow \infty, \operatorname{Im}\left(S_{n}(\sigma) \cap \sigma\right)$ and $\operatorname{Im}\left(S_{n}^{2}(\sigma) \cap \sigma\right)$ both tend to infinity with

$$
\operatorname{Im}\left[S_{n}^{2}(\sigma) \cap \sigma\right]<\operatorname{Im}\left[S_{n}(\sigma) \cap \sigma\right] .
$$

But this leads to a contradiction as follows. The collection of all $S$ with $S(\sigma) \cap \sigma \neq$ $\emptyset$ cannot contain infinitely many elements that are powers of the same primitive element in the group else infinity is fixed by that element. Thus the elements $S$ with $S(\sigma) \cap \sigma \neq \emptyset$ comprise a countably infinite collection of finite sets. These are of the form $\left\{T^{n_{1}}, T^{n_{2}}, \ldots T^{n_{k}}\right\}$ where $T \in \Gamma$ listed in order of increasing height of the point of intersection. However, the first intersection points from these sets have heights increasing to infinity and this contradicts (3.5) above. With this, the proof of the theorem is completed.

We have seen that any point of $D \cup G$ is represented on the boundary of some convex, locally finite fundamental domain. The only other points which could possibly be so represented must lie in $H$ (the horocyclic limit set) but not in the conical limit set. Pommerenke [5] has shown that this set can have full measure. Our next two results show that both simple and non simple points can lie in $H$ but not in the conical limit set.

Theorem 3.3. There exists a Fuchsian group $G$ and a convex, locally finite fundamental domain for $G$ which has a horocyclic limit point on its boundary.

Proof. Let $S, T$ be fractional linear transforms preserving the upper halfplane defined by

$$
S(z)=16 z, \quad T(z)=\frac{7 z+40}{2 z+23} .
$$

Let $\Gamma$ be the group generated by $S$ and $T$. Define four circles $C_{S}, C_{S^{-1}}, C_{T}$, $C_{T^{-1}}$ as follows. Each circle is centered on the real axis; $C_{T^{-1}}$ meets the axis at 1 and $10 ; C_{T^{-1}}$ meets the axis at $-17 / 5$ and $-190 / 13 ; C_{S}$ meets the axis at $-15 / 16$ and $15 / 16 ; C_{S}$ at -15 and 15 . Note that $T$ maps $C_{T}$ onto $C_{T^{-1}}$ and the exterior of $C_{T}$ onto the interior of $C_{T^{-1}}$. Similarly $S$ maps $C_{S}$ onto $C_{S^{-1}}$. The situation is illustrated below.

The region $F$ indicated in the diagram is a convex, locally finite fundamental domain for the discrete group $\Gamma$ generated by $S$ and $T$. In fact $\Gamma$ is a Schottky group with $F$ a Schottky domain. We define

$$
\Omega=\bigcup_{n=-\infty}^{\infty} S^{n}(F)
$$




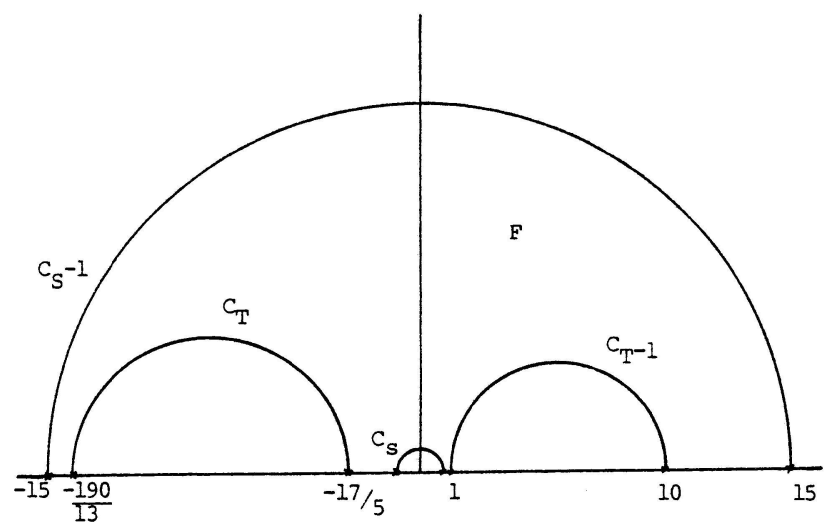

and note that it is a Schottky domain for the group

$$
G=\left\langle\left\{S^{n} T S^{-n}\right\}\right\rangle \text {. }
$$

The point at $\infty$ is on the boundary of $\Omega$. Now we note that

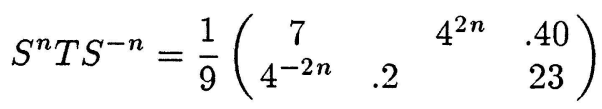

and, from Lemma 3.1, it follows that $\infty$ is a horocylic limit point. This completes the proof of the theorem.

We conclude this section by giving an example of a horocyclic, non-conical limit point which is non-simple.

Theorem 3.4. There exists a Fuchsian group $\Gamma$ and a horocyclic, non-conical limit point for $\Gamma$ which is also non-simple.

Proof. We utilize the same "building block" as in Theorem 2.5 to construct a surface with a geodesic $\sigma$ each half of which crosses itself infinitely often yet eventually leaves any compact set. It then follows from Theorems 2.1 and 3.2 that if we lift $\sigma$ to a geodesic in the upper half-plane its end points will be horocyclic, non-conical, limit points.

Glue copies of the "building block" to obtain the surface and geodesic $\sigma$ on the next page:

\section{Concluding remarks}

In contrast to the situation in 2-dimensions, most geodesics in hyperbolic $n$-space $H^{n}$ with $n \geq 3$ are simple. However, if one attemps the construction of Theorem 2.4 one obtains a domain which is, in general, not convex.

Acknowledgements. We would like to thank Scott Wolpert for his helpful comments on simple geodesics. 


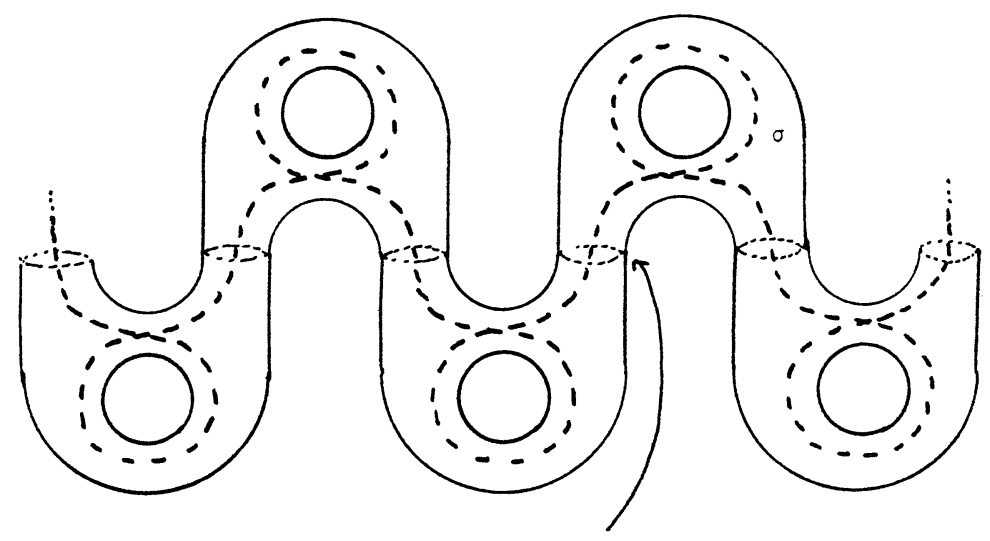

Boundary curves of building blocks

\section{References}

[1] Beardon, A.F.: The geometry of discrete groups. - Springer-Verlag, New York, 1983.

[2] MASkit, B.: Kleinian groups. - Springer-Verlag, Berlin, 1988.

[3] Nicholls, P.J.: Garnett points for Fuchsian groups. - Bull. London Math. Soc. 12, 1980, 216-218.

[4] Nicholls, P.J.: The limit set of a discrete group of hyperbolic motions. - In Holomorphic functions and moduli II, edited by D. Drasin et al., Springer-Verlag, New York, 1988.

[5] Pommerenke, Ch.: On the Green's function of Fuchsian groups. - Ann. Acad. Sci. Fenn. Ser. A I Math. 2, 1976, 409-427.

[6] Sullivan, D.: On the ergodic theory at infinity of an arbitrary discrete group of hyperbolic motions. - In Riemann surfaces and related topics: Proceedings of the 1978 Stony Brook Conference, edited by I. Kra and B. Maskit, Ann. of Math. Studies 97, 1981, Princeton University Press, Princeton, 465-496.

Northern Illinois University

Department of Mathematical Sciences

DeKalb, IL 60115

U.S.A.
Northern Illinois University

Department of Mathematical Sciences

DeKalb, IL 60115

U.S.A.

Received 22 November 1988 